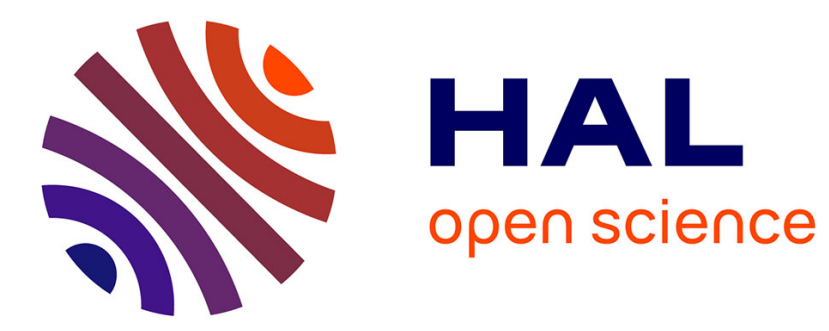

\title{
Polyvalence des toponymes et interprétation en contexte
}

Michelle Lecolle

\section{To cite this version:}

Michelle Lecolle. Polyvalence des toponymes et interprétation en contexte. Pratiques: linguistique, littérature, didactique, 2006, Textes - Contextes, 129/130, pp.107-122. halshs-00304134

\section{HAL Id: halshs-00304134 https://shs.hal.science/halshs-00304134}

Submitted on 24 Aug 2016

HAL is a multi-disciplinary open access archive for the deposit and dissemination of scientific research documents, whether they are published or not. The documents may come from teaching and research institutions in France or abroad, or from public or private research centers.
L'archive ouverte pluridisciplinaire HAL, est destinée au dépôt et à la diffusion de documents scientifiques de niveau recherche, publiés ou non, émanant des établissements d'enseignement et de recherche français ou étrangers, des laboratoires publics ou privés. 


\title{
Polyvalence des toponymes et interprétation en contexte
}

\author{
Michelle Lecolle, Université de Metz, CELTED
}

Il peut paraître étonnant qu'un article portant sur la sémantique des toponymes prenne place dans un numéro consacré à la question du texte et du contexte. En effet, le toponyme, envisagé sur le modèle de l'ensemble des noms propres (Npr désormais), est justement supposé univoque et invariable quel que soit le contexte.

Pourtant, si l'on s'attache à décrire l'interprétation de diverses réalisations de toponymes, parmi lesquelles les réalisations généralement qualifiées de métonymiques, une réalité beaucoup plus diverse apparaît. Ainsi, pour ne citer que quelques catégories de valeurs sémanticoréférentielles du toponyme déjà répertoriées ailleurs (Lecolle 2003 et 2004), celui-ci peut désigner l'ensemble des habitants d'un lieu, comme en (1):

(1) Paris a froid. Paris a faim. (Eluard, Au rendez-vous allemand, 1942).

Il peut prendre un sens événementiel, comme en (2) :

(2) Un millier d'experts internationaux sont réunis cette semaine à Vienne pour faire le point sur les conséquences sanitaires et socio-économiques de Tchernobyl. (Le Monde, 1995-1996)

Lorsqu'il s'agit d'un nom de capitale comme en (3), il peut désigner une instance institutionnelle (pays, gouvernement, gouvernant) :

(3) Au deuxième jour de l'opération militaire contre les forces serbes en Yougoslavie, Washington annonce une intensification des bombardements. (Le Figaro, 1999)

En outre, ces différentes valeurs sont parfois amenées à se faire concurrence, comme on peut l'observer en (4) où, malgré les apparences, Washington dans son contexte ne renvoie ni au gouvernement ni aux habitants ni à la ville elle-même mais à l'événement /réunion des ministres des finances du G7 du 27 septembre 2002/.

(4) 200000 manifestants anti-mondialisation veulent paralyser Washington. (Le Monde, 2002 en titre).

Enfin, certaines de ces valeurs peuvent également se combiner ou encore demeurer indistinctes, selon un modèle respectivement de surdétermination (cumul de sens) et de neutralisation, où l'alternative entre deux interprétations est “ dépassée au profit d'une signification intermédiaire ou indistincte" (Fuchs 1991).

Loin d'être immuables et univoques, les occurrences de toponymes nécessitent par conséquent une interprétation spécifique, largement tributaire du contexte. Sur cette base où le rapport occurrence/référent et la catégorie sémantique du nom ne sont nullement fixes, nous parlerons de construction du sens et de la référence des toponymes en contexte plutôt que de "reconnaissance" ou "d'assignation" du sens et de la référence. Précisons toutefois que parler de "construction" ne revient pas à prôner une approche dite " constructiviste". En effet, pour nous, Tchernobyl, avant d'être un nom d'événement, a référé/réfère bien à un lieu, ce 
que nous constatons d'ailleurs nettement dans nos corpus ${ }^{1}$. Mais la métonymie joue et rejoue le rapport du Npr à son référent initial, et ouvre ainsi un espace d'interprétation.

Cette construction du sens et de la référence des toponymes ne concerne pourtant pas uniquement les sens dérivés - métonymiques. Elle concerne aussi, et fondamentalement, la polyvalence intrinsèque de certains toponymes, et renvoie notamment, pour les noms de pays (Npays), à ce que Cislaru (2005) considère comme l'hybridité de leur sens conceptuel - lieu et pays, le lieu supposant une valeur topologique et des habitants, le pays supposant notamment une valeur institutionnelle, des gouvernants et des citoyens ${ }^{2}$.

Au-delà, une polyvalence comparable - et par voie de conséquence une possible plurivocité comparable - peut être relevée pour d'autres catégories de noms : citons notamment les noms communs $(\mathrm{Nc})$ d'action ou d'activité tels que production, sélection, susceptibles, lorsqu'ils sont en emploi actanciel ou résultatif, de référer à des ensembles - de prendre une valeur collective donc - et de le faire de manière extrêmement flexible, en dépendance étroite avec leur contexte. Et le cas enfin des noms collectifs lexicaux (Ncoll), et en particulier des Ncolls institutionnels (le gouvernement), qui peuvent être interprétés de manière collective (référence au tout) et distributive (référence aux éléments) ${ }^{3}$. Autant de cas où il nous parait légitime de parler de "construction" du sens (quelle est la catégorie sémantique pertinente ?) et de la référence (de quoi, de qui s'agit-il ?).

L'ouverture d'un espace d'interprétation, due à la polyvalence intrinsèque des toponymes et à leurs emplois métonymiques, conduit à réexaminer finement la question de l'ambiguïté (du cumul de sens, de l'indétermination) et le rôle du contexte. En effet, ces emplois, tout en maintenant l'unicité du référent, rejouent, selon des modes divers, la "rigidité " du rapport référentiel et confèrent au toponyme épaisseur sémantique et complexité interprétative. On aboutit ainsi à l'apparent paradoxe : tout en restant désignateur d'un individu, le Npr présente, de par sa polyvalence, ou acquiert, par métonymie, une plurivocité : il peut désigner plusieurs individus (l'individu-lieu, l'individu-ville, l'individu-événement, l'individu pluriel des habitants).

Le propos de cet article est tout d'abord de baliser les différents types de plurivocité présentées par les toponymes ${ }^{4}$. Dans ce cadre, l'ambiguïté - " alternative entre [...] deux significations mutuellement exclusives" (Fuchs 1991: 108) - n'est qu'un cas particulier. Après la présentation de notre corpus, notre première partie détaillera les différentes modalités de cette plurivocité, aux niveaux sémantique (catégorie sémantique de l'occurrence du Npr) et référentiel.

Dans une deuxième partie, nous commencerons par examiner le rôle que peut jouer le " contenu " du Npr (Gary-Prieur 1994) sur l'interprétation des toponymes dans différentes valeurs, avant d'aborder l'influence du contexte - des contextes - sur le maintien ou non de la plurivocité. Comme il apparaitra dans notre développement, le point de vue général qui sous-tend

\footnotetext{
${ }^{1}$ En revanche, si Outreau par exemple est bien une ville, la référence de Outreau telle qu'elle est donnée à voir dans les discours circulants notamment en 2005-2006 (Outreau : “ procès-catastrophe ”, Outreau : " erreur judiciaire ", " fiasco judiciaire "...), s'est largement émancipée de cette base locative.

${ }^{2}$ Cette hybridité peut être retrouvée d'ailleurs dans les noms de villes, si l'on envisage le fait que, outre des habitants, celles-ci possèdent également des instances dirigeantes (communales). Au niveau de description où nous nous situons ici, nous considérerons les deux types de toponymes comme équivalents du point de vue de leur hybridité, sans pour autant nier la spécificité par ailleurs des Npays, bien montrée par Cislaru.

${ }^{3}$ Ceci n'est pas sans rapport avec la question des toponymes : nous aurons en effet à intégrer les Ncolls dans notre analyse de certains toponymes métonymiques.

${ }^{4}$ Nous ne nous attacherons pas ici à distinguer les emplois métonymiques des autres, préférant souligner la polyvalence plus générale des toponymes.
} 
notre appréhension du contexte est celui d'une interaction dynamique des différents paliers que sont le syntagme, la phrase, le texte et l'intertexte. C'est pourquoi nous ne distinguerons pas ici co-texte et contexte, comme il est fait usuellement, et conserverons le terme générique de "contexte" que nous préciserons au fil de l'analyse.

\section{Corpus}

Nous rassemblons pour la présente étude plusieurs corpus, collectés pour les différents travaux présentés en bibliographie. Ils ont en commun de provenir de la presse écrite (écrits de journalistes ou de collaborateurs occasionnels - chercheurs ou hommes politiques dans des tribunes). Il s'agit donc de textes relativement courts centrés sur des événements, ou de dossiers thématiques (le festival de Cannes 2001, la guerre du Kosovo en 1999, différents forums sociaux - Mumbay (Bombay), Porto Alegre, et Bamako en 2006 notamment) ${ }^{5}$. Nous y ajoutons des exemples collectés plus ponctuellement.

Comme nous l'avons défendu dans un précédent article (Lecolle à paraître), nous considérons le corpus comme un échantillon de langage dans lequel s'interprètent les occurrences de toponymes, mais aussi dans lequel se construit un savoir sur les objets référés par le biais des prédications dans lesquelles entrent les Npr considérés. Dans ce cadre, le contexte pertinent, celui dont nous tenons nos connaissances et interprétations des Npr, correspond au texte lui-même avec son paratexte (titraille); à l'intertexte que sont les textes mis en présence dans une même thématique et dans un cadre temporel circonscrit - nous distinguons donc plusieurs souscorpus thématiques, qui peuvent prescrire, à leur manière, des interprétations différentes. Enfin nous tirons également parti des valeurs sémantico-référentielles métonymiques stabilisées des Npr, relevant d'un univers interdiscursif ${ }^{6}$, telles que Tchernobyl (événement) ou les Npr institutionnels comme Matignon, l'Élysée et les noms de capitales. Leur interprétation relève d'une intégration en compétence discursive, basée sur des références partagées.

\section{Toponymes : une valeur sémantique à tiroirs}

La métonymie mais aussi la polyvalence des toponymes eux-mêmes sont susceptibles de démultiplier les cas de plurivocité selon les strates du sens et de la référence que nous précisons dans ce qui suit.

\subsection{Sens des toponymes et types de plurivocité}

Dans un travail précédent (Lecolle 2004), nous avons répertorié différents types de plurivocité du toponyme en nous attachant aux valeurs sémantiques des occurrences elles-mêmes. Nous avons ainsi distingué une "polyréférentialité externe "7, selon laquelle le toponyme, comme

\footnotetext{
${ }^{5}$ Cf. la rubrique " corpus " en fin d'article.

${ }^{6}$ Nous entendons par interdiscours l'ensemble des discours, non attestés dans notre corpus, mais qui “ ont eu un support textuel [...] dont on n'a pas mémorisé la configuration "(Dictionnaire d'analyse du discours p. 325).

${ }^{7}$ Nous avons parlé de "polyréférentialité ", dans le souci de ne pas nous engager sur la question complexe du

" sens" du Npr. Pourtant, on peut distinguer un niveau de " sens" - sens locatif, sens événementiel, sens institutionnel, sens /habitants/ - qui correspond à un trait catégoriel et présente généralement une contrepartie syntaxique. Par voie de conséquence, il conviendrait peut-être alors de parler de " polysémie " plutôt que de " polyréférentialité ". Puisqu'il faut trancher, nous maintenons pourtant ce dernier terme, considérant, comme Cislaru (op. cit.), qu'on a affaire avec le toponyme à un complexe sémantico-référentiel.
} 
rappelé plus haut, peut avoir plusieurs sens mutuellement exclusifs, et une " polyréférentialité interne " où les sens se cumulent sur le mode de l'ambivalence ${ }^{8}$ ou de la surdétermination.

Selon la "polyréférentialité externe", le nom Cannes par exemple renvoie, dans un corpus thématique portant sur le festival, concurrentiellement à la ville de Cannes et au festival luimême (interprétation événementielle), mais aussi, dans un autre corpus thématique portant sur la politique européenne, à la ville (lieu), la ville (institution) et au sommet européen qui s'y est tenu (événement) :

(5) [Jacques Chirac] entend que Cannes donne une "impulsion " au programme de grands travaux [...] communautaires déjà décidés par les Quinze. (Le Monde, 1995-1996)

Mais les différents sens d'un même toponyme peuvent aussi se rencontrer. Ils doivent alors être discriminés, sur le mode de l'ambiguïté stricto sensu, comme en (6) où un choix s'impose entre l'interprétation/habitants/ et l'interprétation /pays/ :

(6) Le Japon épargne. (France Inter, 2001)

ou en (7) où le sens de Cannes (lieu ou festival) reste indécidable :

(7) Coppola a gardé d'excellents souvenirs de Cannes. (Le Monde, 1995-1996)

Selon la "polyréférentialité interne" d'un autre côté, le sens d'une occurrence est compositionnel. Ainsi, en (8), la syntaxe impose un sens locatif (/place Tiananmen/), mais le sens réel de l'occurrence nécessite à nos yeux de prendre en compte conjointement la valeur événementielle qu'a acquise Tiananmen :

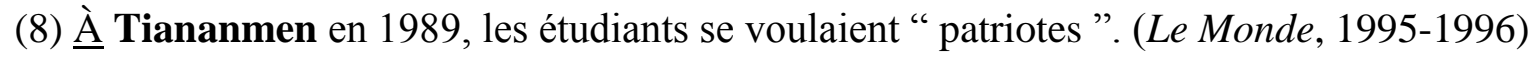

de même qu'on ne peut réduire, en (9), Atlanta à une interprétation purement locative :

(9) Selon l'agence japonaise Kyodo, ce dirigeant a fait allusion à la controverse qui avait entouré, en 1994, le projet du président taïwanais Lee Teng-hui d'assister aux Jeux asiatiques d'Hiroshima. "La Chine réagira avec détermination, si quelque chose comme cela devait se produire à Atlanta ", a averti M. Shaozhu. (Le Monde, 1995-1996)

et encore moins Outreau, en $(10)^{9}$ :

(10) Fabienne Roy Nansion [avocate, entendue par la Commission parlementaire constituée en décembre 2005 à la suite de " l'affaire d'Outreau "] s'arrête : "Nous sommes les rescapés d'une tornade noire qui s'est abattue sur Outreau. Nous étions dans l'oeil du cyclone, là où on ne voit rien." (Libération, 02/2006)

Le même type de cumul peut être constaté entre sens géographique et institutionnel du Npays et sens /habitants/ en (11) où le Npays la Russie renvoie au pays, mais aussi à sa population, puisque l'ensemble du texte porte sur les réactions du peuple russe à la guerre du Kosovo :

(11) On prend en exemple l'expansion de l'OTAN vers les frontières d'une Russie qui ne se remet pas d'avoir perdu la guerre froide. (Le Figaro, 1999. En titre : A Moscou, le " parti de la guerre" se mobilise).

\footnotetext{
${ }^{8}$ Le terme " ambivalence " est dû à Le Goffic (1982) et correspond au cumul de sens.

${ }^{9}$ De fait, Outreau est proprement inclassable d'un point de vue sémantico-référentiel ...
} 
Enfin, le titre (12) joue sur les valeurs locative et institutionnelle du Npr de capitale Bamako, et sur la référence allusive au sens événementiel (alter renvoyant à " altermondialisme ”, donc à "Forum social ") :

(12) Bamako, capitale alter (Libération, 01/2006, titre d'un article sur le Forum Social de Bamako).

D'autres combinaisons relevant de la polyréférentialité interne peuvent être observées (cf. Lecolle 2004), d'où il ressort que cette compositionnalité est loin d'être marginale ${ }^{10}$.

Nous tirons de ce qui précède un enseignement: le modèle de l'homonymie et de l'ambiguïté ${ }^{11}$ du Npr en contexte, généralement adopté par la tradition logico-grammaticale et reprise notamment dans le cadre du Traitement Automatique de la Langue, est réducteur en ce qu'il présente comme discrètes et disjointes des valeurs sémantiques qui gagnent à être analysées sur le mode du continu. Cette réduction à ce que nous nommons ici " polyréférentialité externe " tient sans doute à ce que, d'une part la plupart des travaux sur le Npr s'intéressent prioritairement à l'anthroponyme, oubliant par là même la spécificité des toponymes - leur polyvalence intrinsèque -, et d'autre part au fait qu'est généralement laissé de côté le cas de la métonymie qui met précisément en relation plusieurs valeurs - le sens " propre ", le sens métonymique, mais aussi leur combinaison -, et ceci plutôt sur le mode de la composition que sur celui de la substitution, celle-ci n'étant qu'une éventualité parmi d'autres.

La polyvalence, mais aussi la labilité de l'interprétation se retrouvent à un second niveau, celui de la référence du toponyme en contexte.

\subsection{Flexibilité référentielle-indétermination référentielle}

Le sens et la référence d'un toponyme peuvent être interprétés conjointement dans plusieurs des cas cités plus haut : ainsi, dans un contexte général traitant du festival de Cannes, assigner, sur la base d'indices syntaxiques - par exemple une préposition temporelle -, un sens événementiel à Cannes, revient ipso facto à lui attribuer la référence " festival " et telle occurrence annuelle de festival, sur la base d'autres éléments contextuels (cf. § 3.3.2). De même, le sens locatif de Cannes trouve une correspondance référentielle directe. De son côté, le sens collectif /habitants/ d'un toponyme correspond référentiellement à une référence unique - un individu collectif. Et la plurivocité du toponyme rendue plus haut sous les termes de "polyréférentialité externe et interne " se retrouve alors également au niveau du/des référents en jeu.

Il n'en est pas de même, pourtant, pour les toponymes à valeur métonymique institutionnelle (noms de capitale, nom de lieux institutionnels - Matignon, Washington), où les "strates" sémantique et référentielle n'entrent pas aussi simplement en correspondance. En effet, un sens institutionnel constant peut permettre à ces toponymes de référer à une collectivité (l'institution-gouvernement, les membres de l'institution) ou à un individu (le premier ministre, le président américain). Le plus souvent, ces différences sont neutralisées et le référent est alors sous-spécifié (indéterminé). A l'instar de certains Ncolls, les toponymes institutionnels possèdent ainsi un caractère continu interne.

Mais à une occurrence de Npr institutionnel peut également correspondre un référent nettement plus circonscrit. Ainsi, dans un corpus portant sur un événement de politique européenne, Vienne réfère systématiquement au pays (l'Autriche) et non au gouvernement :

\footnotetext{
${ }^{10}$ Cf. également Cislaru op. cit. (pp. 258-259) pour des conclusions similaires à propos des Npays.

${ }^{11}$ Cf. Fuchs art. cit. p. 110.
} 
(13) La menace européenne d'isoler le pays en cas de participation du parti de Jörg Haider au gouvernement inquiète les Autrichiens. Mais Bruxelles n'a pas les moyens juridiques d'exclure Vienne de l'Union. (Libération, 2000)

Et dans une information concernant les élections régionales de 2004, 1'Elysée et Matignon paraissent bien référer aux personnes correspondantes :

(14) L'Élysée et Matignon commencent à se faire des cheveux. (France Culture, le 22/03/04)

On le voit à travers ces exemples, les métonymies institutionnelles possèdent une certaine flexibilité : l'éventail des interprétations référentielles d'un toponyme dont le sens est institutionnel se situe donc entre, d'un côté, une interprétation précise - personne (dirigeant) ou groupe de personnes ou institution (pays) -, et, d'un autre côté, une interprétation qui ne distingue pas ces différents objets, i.e. qui neutralise les différences. Il y a donc ici deux niveaux d'indétermination et d'adaptation interprétative : (i) celle qui est liée en quelque sorte à la précision d'ajustement nécessaire et (ii) l'indétermination tenant à la composition de l'ensemble référé. L'interprétabilité de ces emplois métonymiques en termes institutionnels demeure néanmoins garantie par leur stéréotypie formelle en discours journalistique - on peut parler de routine sociolectale. C'est pour souligner la conjugaison de cette interprétabilité sémantique et de cette souplesse dénotative que nous avons qualifié ceux-ci de "noms joker" (Lecolle 2003).

\section{Contexte, contextes}

Comme il apparaît déjà dans le dernier paragraphe, il va sans dire que le contexte intervient de manière cruciale dans l'interprétation des toponymes : à partir de la polyvalence décrite, il peut maintenir la plurivocité ou l'indétermination référentielle, ou au contraire, selon les prescriptions conjointes des différents paliers présentés en introduction, apporter des spécifications suffisantes pour amener univocité et détermination.

\subsection{Ambiguïté et " filtrage contextuel"}

Une position de principe tout d'abord : nous ne pensons pas que le contexte désambiguïse nécessairement ce qui est ambigu, détermine ce qui est indéterminé, et encore moins ramène à l'univocité ce qui est plurivoque. Nous ne considérons d'ailleurs cette pluralité de valeurs ni forcément comme un problème (pour la communication par exemple) ni comme une exception. Par ailleurs, comme nous l'avons posé en introduction, les valeurs sémanticoréférentielles des Npr, en particulier lorsqu'ils sont métonymisés, ne leur sont pas assignées $a$ priori mais doivent se construire. C'est pourquoi à une conception du contexte comme "filtre"12, qui "désambiguïserait" et ainsi dégagerait la bonne valeur sémanticoréférentielle, nous préférons le point de vue d'un contexte qui construit, et l'idée d'une coconstruction d'éléments qui, pris isolément, pourraient demeurer obscurs. Un exemple concernant l'interprétation des Npr développé dans Lecolle (à paraître) illustrera cette affirmation : pour interpréter Cannes en (15) comme événementiel (le festival), nul n'est besoin de connaître Kumashiro et Appassionata. Il suffit d'interpréter les Npr comme référant à des actants prototypiques (réalisateur et film) de l'événement festival :

\footnotetext{
${ }^{12}$ Cf. la critique du filtrage contextuel, notamment chez Fuchs (art. cit.).
} 
(15) Après le déclin du roman-porno, Kumashiro sera encore remarqué pour $\underline{\text { Appassionata }}^{13}$, présenté à Cannes en 1983. (Le Monde, 1995-1996)

Qui plus est, on est amené ici à acquérir une connaissance, fût-elle minimale, du " contenu " de ces Npr, chacun d'entre eux venant en quelque sorte en appui des autres.

\subsection{Contexte et " contenu" du Npr}

Nous empruntons la notion de "contenu" du Npr à Gary-Prieur (1994), qui la présente comme "les propriétés qui caractérisent le nom propre en tant qu'il est lié à son référent initial " (p. 40). Plus loin l'auteur parle du contenu comme de l'ensemble des traits encyclopédiques attachés au référent du Npr permettant de comprendre les énoncés contenant ce nom (p. 46) - un sous-ensemble des traits encyclopédiques, sélectionnés par le contexte, donc. Mais si la "connaissance discursive" du Np, comme préalable, est bien celle qui est nécessaire à la compréhension du texte et de l'occurrence du Npr, on peut aussi considérer qu'elle est apportée par le texte ${ }^{14}$. C'est dans cet esprit que nous avons repris la notion de "contenu du Npr", dans l'article cité ci-dessus, cherchant à délimiter comment cette connaissance est utilisée pour le repérage et l'interprétation de toponymes à sens événementiel, mais aussi à observer comment le contenu se construit. Le cas d'un toponyme employé métonymiquement dans un sens événementiel est à cet égard particulièrement instructif puisqu'il est candidat à entrer en quelque sorte dans un "baptême" second, celui d'un événement ${ }^{15}$.

Les observations succinctes que nous avons développées précédemment peuvent être exploitées et poursuivies dans deux directions au moins : d'une part, le contexte, qui, dans l'approche de Gary-Prieur, "filtre " les informations pertinentes - ce à quoi nous souscrivons dans une certaine mesure, cf. ci-dessous -, est aussi celui qui pose et développe d'autres informations sur le Npr, dans un processus cumulatif. D'autre part, la question du contenu, qui concerne le référent du Npr, n'est pas sans incidence sur son sens, ce qui est particulièrement manifeste dans le cas des antonomases, qui exploitent la typicité du référent $\mathrm{du} \mathrm{Npr}-l e$ Cannes de la vidéo - mais aussi des comparaisons et sériations - (16) et (17) :

(16) Mumbai, antithèse de Porto Alegre à bien des égards. (Politis, 15/01/2004)

(17) Ce n'est pas Tchernobyl, loin de là, mais la pollution nucléaire survenue en 1993 à Pierrelatte (Drôme) [...] en dit long sur le flou qui peut entourer les opérations d'assainissement et de décontamination radioactive. (Le Monde, 1995-1996)

De même, c'est la connaissance du contenu de Tiananmen, rassemblé au niveau textuel et surtout intertextuel et interdiscursif, qui permettait de repérer en (8) une plurivocité, mais aussi d'interpréter la phrase - les étudiants notamment appartenant, en tant qu'actants, au scénario de l'événement Tiananmen. Si le scénario - ce que nous avons pu nommer " les constituants" (actants ou parties) de l'événement - est attaché au toponyme dans sa valeur événementielle, il faut souligner également que certaines informations liées au référent initial du toponyme lui-même peuvent être engagées dans l'interprétation : ainsi il n'est sans doute pas indifférent

\footnotetext{
${ }^{13}$ Italiques du texte initial, généralement adoptées pour citer des oeuvres : elles nous apportent un élément d'interprétation.

${ }^{14}$ La perspective de Gary-Prieur est essentiellement attachée au palier de l'énoncé. Pour notre part, nous pensons qu'il est loisible de passer de l'énoncé au texte. Nous étendons également la notion de " contenu " au-delà de la proposition initiale, comme il apparaîtra ici.

${ }^{15}$ Ce second baptême serait cependant d'un autre type que le premier, puisque le sens (et surtout le contenu) premiers du nom de lieu peuvent se composer avec le sens et contenu événementiels (cf. infra).
} 
dans certains contextes de savoir que le lieu Tchernobyl se trouve en Ukraine (ancien bloc soviétique), que Outreau est une ville située au bord de la mer - cf. (19) ci-dessous -, dans le Nord de la France. Remarquons d'ailleurs que le transfert du contenu du Npr sur ses valeurs métonymiques est susceptible d'intervenir aussi dans le cas des Npr d'auteurs : les connaissances attachées à la personne d'un auteur peuvent avoir une influence sur le sens d'une occurrence métonymique de son nom (faire du Proust) et, réciproquement, les discours construits à propos de l'œuvre peuvent nous renseigner sur l'auteur lui-même ${ }^{16}$. On retiendra donc ici que, à travers des parcours textuels et discursifs, le Npr, tout en conservant (et parce qu'il conserve) sa valeur de désignateur d'un individu, gagne en épaisseur sémantico-référentielle, ce qui explique dans une large mesure l'interprétabilité des différents types métonymiques possibles, les cumuls de sens mais aussi les allusions culturelles. C'est ainsi que nous expliquons (16) cité ci-dessus, et, dans le même texte :

(18) De Porto Alegre à Bombay (Mumbay), il y a bien plus qu'une demi-révolution de globe.

Et, en (19), la syllepse (figure du double sens) sur les sables mouvants d'Outreau, où la polyréférentialité interne de Outreau est exhibée, tirant parti du contenu du toponyme pour forger la métaphore les sables mouvants :

(19) Pour la première fois, les députés vont être entraînés dans les sables mouvants d'Outreau. (Libération, 02/2006, à propos de l'enquête parlementaire menée début 2006 à la suite $\mathrm{du}$ "fiasco " d'Outreau)

Il ne s'agit naturellement pas ici de soutenir que l'ensemble des connaissances encyclopédiques attachées au Npr (le faisceau de ses propriétés) est mobilisé à chacune de ses occurrences. Mais de tenter d'expliquer la variété des valeurs du toponyme selon les textes, et surtout la souplesse avec laquelle il glisse de l'une à l'autre. Ainsi, dans les textes de Politis concernant les Forums Sociaux que nous avons analysés, le contenu du Npr initial (le lieu) est largement thématisé, puisqu'il sert l'analyse et la présentation comparative des différents forums selon les changements qu'induit le changement de continent. On voit aussi à travers les exemples de ces textes - (16) et (18) - que ce contenu sert à construire sémantiquement et grammaticalement la polyréférentialité de l'occurrence du toponyme - syntaxe locative et interprétation plurielle. Ce n'est bien sûr pas le cas de tous les textes et de tous les emplois de toponymes : le contexte intervient également comme filtre, ramenant alors le toponyme à une seule valeur (cf. par exemple l'article de Libération du 9 février 2006 titré A Outreau, silence et agacement, qui porte sur la ville elle-même et ses habitants, et laisse au second plan - mais présupposé - l'événement lui-même).

Dans ce qui suit, nous envisagerons les types de contexte (phrastique, textuel, intertextuel) considérés non pas isolément et par strates emboîtées, mais de manière interactive, en tirant parti des observations qui précèdent.

\subsection{Contexte, univocité et plurivocité des toponymes}

Il ressort de ce qui précède que, si l'on tient compte du contenu d'un toponyme, stabilisé sur des bases que nous nommerons très largement “interdiscursives ${ }^{17}$ ", l'interprétation phrastique fondée sur des indices distributionnels est insuffisante, voire trompeuse - nous l'avons

\footnotetext{
${ }^{16}$ Dans le cas des métonymies auteur/œuvre, on peut d'ailleurs se demander si le nom de l'auteur est réellement "premier" : que vaut en effet ce nom indépendamment de la notoriété due à l'œuvre ?

${ }^{17}$ Relevant de l'intertexte stricto sensu ou de connaissances discursives stabilisées.
} 
déjà constaté précédemment avec la syntaxe locative. De fait, elle est plutôt complétée ou rejouée par des éléments relevant d'autres paliers contextuels.

\subsubsection{Univocité des toponymes : apport et limite des indices distributionnels}

Les indices phrastiques, syntaxiques, et surtout lexicaux, interviennent cependant dans certains cas dans le sens de la spécification d'une valeur du toponyme : nous l'avons constaté dans un travail portant sur l'interprétation événementielle des toponymes Tchernobyl, Tiananmen, Atlanta et Cannes ${ }^{18}$.

Nous avons ainsi pu répertorier, sur des bases phrastiques, les différents sens que prend Tchernobyl dans Le Monde 1995-1996. Le tableau suivant nous montre que la valeur événementielle du Npr, pourtant largement stabilisée dans notre compétence discursive, n'est pas majoritaire. On trouve ainsi Tchernobyl dans un sens locatif :

(20) Évacués de Pripiat et Tchernobyl [...], les 6500 employés et leurs familles ont été relogés à Slavoutitch, une cité construite spécialement pour eux à 50 kilomètres du site.

et dans le sens de /centrale/ :

(21) On peut en douter, si l'on songe que même Tchernobyl continue à tourner, à côté de son sarcophage.

\begin{tabular}{|l|l|c|c|}
\hline Nom propre & Interprétation & Occurrences & Pourcentage \\
\hline Tchernobyl & événement & 134 & $39,88 \%$ \\
\hline Tchernobyl & lieu & 121 & $36,01 \%$ \\
\hline Tchernobyl & lieu et événement (polyréférentialité interne) & 6 & $1,79 \%$ \\
\hline Tchernobyl & “centrale ”: Tchernobyl est le nom de la centrale & 68 & $20,24 \%$ \\
\hline Tchernobyl & $?$ & 7 & $2,08 \%$ \\
\hline & & $\mathbf{3 3 6}$ & $100 \%$ \\
\hline
\end{tabular}

\section{Interprétation événementielle}

Quant à la valeur événementielle, elle est basée sur plusieurs types d'indices, parmi lesquels intervient notablement la structure du $\mathrm{SN}$ [N de Tchernobyl] où le nom tête est commémoration ou anniversaire. Une autre catégorie d'indices, lexicaux, correspond à la présence dans la phrase de ce que nous avons nommé plus haut les "constituants de l'événement" - les éléments de son scénario. Le lexique des constituants de chaque événement lui est spécifique, tout en étant relativement stable et prévisible. Dans le cas de Tchernobyl, les constituants tendent très nettement à situer l'événement - victimes, malades, migrants écologiques - dans le cadre d'une causalité ${ }^{19}$ :

\footnotetext{
${ }^{18}$ Une partie des résultats de ce travail est présentée dans Lecolle (à paraître). Nous avons sélectionné deux événements réputés imprévus (Tchernobyl, Tiananmen) et deux événements prévus (le festival de Cannes, les Jeux Olympiques d'Atlanta).

${ }^{19}$ Comme on peut le remarquer, certains indices sont purement conjoncturels et liés en particulier aux dates des publications (anniversaire). Pourtant, les dimensions historique d'un côté et de causalité/conséquence de l'autre
} 
(22) Des mutations génétiques héréditaires affectent les victimes de Tchernobyl.

Voici, en comparaison quelques éléments du scénario de Cannes (festival) : jury, public, palmarès, palme d'or, film(s)...

D'autres types d'indices, syntaxiques, peuvent conduire à l'interprétation événementielle. En position sujet, ou comme complément d'agent d'une phrase passive, le toponyme a toutes les chances de recevoir l'interprétation "événement". Même si le critère n'est pas toujours discriminant à lui seul (cf. en (24) le lexique : révélé, films), il élimine en tous cas l'interprétation locative du toponyme ${ }^{20}$ :

(23) À l'occasion de sa dernière visite, en novembre 1995, le chancelier a passé en revue un détachement d'infanterie, un geste signifiant que Tiananmen est oublié et pardonné. ( $L e$ Monde, 1995-1996)

(24) Et, parmi les nouveautés, deux “petits films ", également révélés par Cannes, reçoivent eux aussi un bon accueil à l'échelle de leurs ambitions commerciales. (Le Monde, 1995-1996)

Pourtant, les indices lexico-syntaxiques, malgré leur récurrence, trouvent vite leur limite. Ainsi, conséquence sans doute de la polyréférentialité externe des toponymes, les prépositions temporelles après, avant, depuis suivies d'un toponyme ne conduisent à une interprétation événementielle que dans, respectivement, 69,47\%,67,74\% et $50 \%$ des cas (test effectué sur l'ensemble de la base de données, soit 29061 enregistrements). On trouvera ici une valeur fréquente du syntagme prépositionnel en après + toponyme :

(25) Après Londres et Milan, les défilés de l'hiver 1996-1997 se sont tenus à Paris, du 11 au 20 mars. (Le Monde, 1995-1996).

Enfin, une phraséologie récurrente - clichés, routines journalistiques -, constatée à travers l'ensemble des enregistrements analysés, conduit, malgré la syntaxe locative, à une interprétation événementielle de Cannes et Atlanta: (Nfilm, Nacteur, Nsportif) a gagné son ticket/billet pour Cannes/Atlanta ; sur la route d'Atlanta ; le rendez-vous d'Atlanta/Cannes.

Ces apports, mais aussi ces limites nous paraissent confirmer la nécessaire intrication des niveaux d'analyse : la dimension du texte, et au-delà celle du sous-corpus thématique enrichit, oriente, voire détermine les connaissances qui nous permettent d'interpréter une occurrence de toponyme.

\subsubsection{Univocité des toponymes : déterminations textuelles et intertextuelles}

Les thématiques dont relèvent les textes et différents sous-corpus, et parfois les récits qu'ils construisent, sont porteurs de déterminations qui peuvent agir fortement aux niveaux sémantique et référentiel, selon les différentes " strates " présentées plus haut ( $(2)$.

Ainsi, nous avons souligné l'univocité référentielle de Vienne en interprétation institutionnelle - exemple (13), et (26) ci-dessous - dans l'ensemble de notre corpus de politique européenne :

sont extrêmement présentes dans l'entour des toponymes événementiels, en particulier lorsqu'il s'agit d'un événement non prévu (Tchernobyl, Tiananmen). Selon nous, elles sont aussi nettement liées au discours journalistique lui-même (mais c'est une autre histoire ...). Quoi qu'il en soit, ces dimensions, dans des structures syntaxiques diverses, interviennent fortement comme indices de repérage du sens événementiel.

${ }^{20}$ Mais il autorise valeur institutionnelle d'un Npays ou de ville, cf. infra. 
(26) Les Français sont sur la même longueur d'onde que le président de la République [...] quant à l'évolution de la situation politique en Autriche et à l'éventuelle entrée du Parti populiste dans le gouvernement de Vienne. (Le Monde, 2000)

Cette spécificité au regard de la flexibilité référentielle habituelle des métonymies institutionnelles s'explique facilement dans le cadre de la thématique générale du corpus, où ce qui est en cause est précisément la constitution du gouvernement autrichien, pour laquelle l'Autriche se trouve en opposition avec les autres pays de l'Europe (les Quinze).

La thématique des sous-corpus présentant des toponymes à sens événementiel est également discriminante, appuyée par des éléments textuels et phrastiques. Ainsi, la détermination contextuelle en termes de construction du sens et de la référence d'occurrences toponymiques s'observe aisément si l'on compare les différentes thématiques mettant en jeu Cannes comme événement par exemple (sommet européen, festival de cinéma, autre festival). On peut l'observer aussi dans les textes et dossiers portant sur les forums sociaux. Dans ces derniers, interviennent comme interprétants du toponyme plusieurs types d'éléments : une thématique générale centrée sur des problématiques sociales et politiques internationales (migration, environnement) ; la présentation d'actants de l'événement (associations, syndicats, " figures " de l'altermondialisme) ; la mise en série des Npr eux-mêmes (Porto Alegre, Mumbay, Caracas, Bamako, mais aussi Davos), d'où on acquiert parfois des connaissances sur le mode de la coconstruction évoquée plus haut. La reformulation de l'événement sous différentes expressions (le Forum Social Mondial, le forum, le FSM, l'événement, Bamako/Mumbay/Caracas), cf. (27) ci-dessous, prises dans un environnement distributionnel comparable, est susceptible de former un micro-système - Le FSM/Forum Social Mondial/Bombay nous apprendralenseignera/montrera; au programmelen introduction du FSM/Forum Social Mondial/Bombay - qui se construit et se cumule au fil du/des textes.

Ajoutons à ceci la composante tactique du texte (Rastier 2001) - sa syntagmatique - qui, conforme aux prescriptions du discours journalistique, présente généralement en titre l'événement-forum, et le rapporte, en tête d'article (lead), à sa localisation par le toponyme :

(27) Caracas, Bamako, Karachi : la trajectoire, sur la planisphère, des trois villes où se tient cette année le Forum social mondial (FSM) dessine une ligne droite dynamique et croissante. (Politis, 19/01/2006)

et produit en chute le toponyme dans son sens événementiel :

(28) [Bernard Cassen, président d'honneur d'ATTAC]. On observera le devenir du Manifeste [...] qu'il compte bien mettre en avant de Bamako à Caracas.

en passant éventuellement, au fil du texte, par différentes valeurs possibles du toponyme : institutionnel (Bamako en tant que capitale du Mali), population etc.

\subsubsection{Polyvalence des toponymes et glissements de sens}

La détermination du sens et de la référence par le texte trouvent néanmoins des limites : en effet, le sens d'un toponyme peut passer en souplesse d'une valeur à une autre, ce qui d'ailleurs ne doit pas nous étonner, étant donné ce que nous avons pu remarquer précédemment de la polyvalence intrinsèque du toponyme, et de l'intrication de ses différentes valeurs.

Ce glissement s'observe par exemple en (29) où la position sujet des toponymes, appuyée sur nos connaissances (nous sommes en 1999), nous indique que les Npays ont une valeur événementielle -/guerre/. C'est du moins sur ces bases qu'on interprète la Somalie et l'Irak: 
(29) La Somalie a montré que les États-Unis renoncent plus vite que l'Europe, lorsque les cercueils commencent à rentrer au pays. L'Irak a établi l'efficacité douteuse des campagnes aériennes quand il s'agit de faire plier un régime autoritaire. La Bosnie, durablement transformée en protectorat de l'OTAN, prouve qu'il y a loin, très loin, d'un cessez-le-feu au désengagement des troupes américaines. (Le Figaro, 1999).

L'occurrence La Bosnie en revanche illustre une polyréférentialité interne, qui s'exprime syntagmatiquement par le biais d'entours contextuels phrastiques partiellement contradictoires : durablement transformée en protectorat de l'OTAN renvoie au sens institutionnel du Npays, tandis que la fin de la phrase : prouve qu'il y a loin[...] d'un cessez-le-feu au désengagement des troupes américaines, ainsi que le parallélisme sémantique et syntagmatique avec les deux premières phrases orientent vers une interprétation événementielle.

Un glissement de sens comparable s'observait avec l'exemple (11) entre interprétation institutionnelle du Npays la Russie et interprétation /population/ (habitants ou citoyens). Ce glissement (qui n'est d'ailleurs pas sans incidence rhétorique) est, selon nous, favorisé par la polyréférentialité interne de l'occurrence. Plus généralement, la polyvalence des Npays donne lieu à des usages d'une grande flexibilité, telle qu'ils peuvent passer en contexte du sens institutionnel au sens géographique et au sens /habitants/ ou, le plus fréquemment, posséder ces différents sens simultanément, comme en (30) où les verbes (paralyser et, plus encore, gagner) sélectionnent un complément d'objet susceptible de référer à une entité collective :

(30) Comment stopper Haider : par l'ostracisme ou la banalisation ? Cette question qui paralyse l'Autriche depuis des années est en train de gagner l'Allemagne. (Libération, 2000)

C'est ce que reflètent également dans les corpus les nombreuses reprises anaphoriques de Npays par des noms de peuple (la Russie-les Russes) et inversement, présentant ainsi les référents comme interchangeables :

(31) La Russie a présenté jeudi au Conseil de sécurité de l'ONU un projet de résolution [...]. Des propos essentiellement symboliques, la Russie exsangue n'ayant pas les moyens d'une intervention militaire.[...] A Paris, Iouri Loujkov a dénoncé ce que les Russes considèrent comme une "agression ". [...] Ce qui motive l'attitude de la Russie, c'est avant tout un "sentiment d'injustice”. (Le Figaro, 1999).

\section{Conclusion}

Nous nous sommes attachée ici à décrire les différentes valeurs sémantico-référentielles que peuvent prendre des occurrences de toponymes, qu'elles soient ou non métonymiques, et l'incidence du contexte sur leur interprétation. En nous démarquant d'une conception discriminante, "filtrante" du contexte d'une part, et d'une analyse en termes de " désambiguïsation" systématique d'autre part, nous avons pu montrer que le sens et la référence des toponymes, pourtant, comme l'ensemble des Npr, supposés a priori univoques et immuables, sont souvent plurivoques ou indéterminés, et que l'interprétation de leurs occurrences dans les textes doivent être envisagées en termes de construction plutôt qu'en termes de reconnaissance.

Dans cette construction du sens et de la référence - qui, au demeurant, serait à envisager pour les Nc également, lorsqu'ils sont employés de manière indirecte (métonymique par exemple)-, nous avons pris en compte les différents paliers du contexte (du syntagme à l'intertexte d'un sous-corpus composé des textes relevant d'une même thématique), de manière interactive et dynamique. Concernant la composante thématique et son rôle sur 
l'interprétation de $\mathrm{Npr}$, nous avons ainsi pu vérifier dans les faits l'affirmation de Rastier selon laquelle "la détermination du local par le global s'exerce [...] de deux façons, par l'incidence du texte sur ses parties, par l'incidence du corpus sur le texte" (2001: 109). En effet, des déterminations textuelle et intertextuelle peuvent être repérées au sein même de la phrase.

Qui plus est, dans la phrase elle-même, le sens de certains éléments a priori obscurs - des Npr non connus - s'éclairent mutuellement : nous en avons parlé en termes de co-construction. Cette co-construction, due aux rôles simultanément passif et actif du contexte (cf. Rastier op. cit.), apparaît à l'analyse dans diverses structures : mise en relation syntagmatique étroite de réseaux de Npr (énumération, comparaisons et oppositions - Cannes et Berlin ; Porto Alegre, Mumbay et Bamako comme "anti-Davos "), présentation dans le contexte d'un toponyme événementiel des actants prototypiques de son scénario (sportifs de Atlanta, réalisateurs et films de Cannes), eux-mêmes non connus.

Si cette co-construction joue, comme nous l'avons montré, sur l'interprétation en cours des occurrences concernées, elle intervient également dans le processus cumulatif de l'enrichissement de la connaissance du référent du $\mathrm{Npr}$ - ce que nous avons nommé, reprenant le terme de Gary-Prieur (1994), son contenu -, connaissance qui, à son tour, pourra être exploitée pleinement ou réduite (filtrée) dans/par un nouveau contexte. Dans ce processus cumulatif, la polyréférentialité des toponymes, par les cumuls et glissements de sens qu'elle permet, exerce sans doute un rôle non négligeable.

\section{Bibliographie}

Charaudeau P. \& Maingueneau D. (dir.) (2002). Dictionnaire d'analyse du discours. Paris, Seuil.

Cislaru G. (2005). Étude sémantique et discursive du nom de pays dans la presse française avec référence à l'anglais, au roumain et au russe, thèse de doctorat, Université Paris IIISorbonne Nouvelle.

Fuchs C. (1986). “Le vague et l'ambigu : deux frères ennemis". Quaderni di semantica Vol. VII, ${ }^{\circ} 2$, pp. 235-245.

Fuchs C. (1991). "L'hétérogénéité interprétative”. In Parret H. (dir.) Le sens et ses hétérogénéités. Paris, Éditions du CNRS, pp. 107-120.

Gary-Prieur M.-N. (1994). Grammaire du nom propre. Paris, Presses Universitaires de France.

Godard D. \& Jayez J. (1996). “ Types Nominaux et Anaphores : le cas des objets et des événements ". In De Mulder W., Tasmowski-De Ryck L. \& Vetters C. (éds), Anaphores temporelles et (in-)cohérence, Amsterdam et Atlanta : Rodopi, 41-58.

Le Goffic P. (1982). “Ambiguïté et ambivalence en linguistique ”. DRLAV 27, pp. 83-105.

Lecolle M. (2004). “ Toponymes en jeu : Diversité et mixage des emplois métonymiques de toponymes ". Studii si cercetari filologice 3 / 2004, Université de Pitesti, Roumanie, pp. 5-13.

Lecolle M. (à paraître). “Éléments pour la caractérisation des toponymes en emploi événementiel". Actes du Colloque international: "Représentations du sens linguistique III", Bruxelles, du 3 au 5 novembre 2005.

Lecolle M. (2003). Métonymies et figures de référenciation dans la presse écrite généraliste. Analyse sémantique et rhétorique, thèse de doctorat, Université Toulouse-Le Mirail. 
Leroy S. (2004). De l'identification à la catégorisation. L'Antonomase du nom propre en français. Louvain/Paris, Peeters.

Leroy S. (2004). Le nom propre en français. Gap-Paris, Ophrys.

Rastier F. (1998). “Le problème épistémologique du contexte et le statut de l'interprétation dans les sciences du langage". Langages 129, pp. 97-111.

Rastier F. (2001). Arts et sciences du texte. Paris, Presses Universitaires de France.

Searle J.R. (1972). Les actes de langage. Paris, Hermann.

Siblot P. (1999). "Les palimpsestes du texte ou les fantômes de l'interdiscours ". Cahiers de praxématique 13, pp. 113-143.

Vicente Rangel M. (2005). "La glose comme outil de désambiguïsation référentielle des noms propres purs". Corela, Numéros spéciaux, Le traitement lexicographique des noms propres. [en ligne] : http://edel.univ-poitiers.fr/corela/document.php?id=462

\section{Corpus}

Le Monde (1995-1996) sur CD-Rom : 29061 enregistrements phrastiques pré-sélectionnés autour d'une liste d'une trentaine de toponymes (Cannes, Atlanta, Tchernobyl et Tiananmen sont traités ici) et consignés dans une base de données* .

Le Figaro, Le Monde, Libération (1999). Textes portant sur la guerre du Kosovo.

Le Figaro, Le Monde, Libération (2000). Événement de politique européenne.

Le Figaro, Le Monde, Libération (2000). Événement de politique (franco-)française.

Le Figaro, Le Monde, Libération (2001). Festival de Cannes.

Politis, Le Monde, Libération (2004-2006). Textes et dossiers sur différents forums sociaux.

Politis, Le Monde, Libération (janvier-février 2006). Textes et dossiers sur l'affaire d'Outreau.

\footnotetext{
* Les données du CD-Rom ont été traitées à l'aide de Syntex, logiciel d'analyse syntaxique de relations de dépendance. Cet outil a été réalisé par Didier Bourigault à l'ERSS, Toulouse. Nous remercions celui-ci pour l'aide qu'il nous a apportée dans ce travail.
} 